\section{miRNAs target the flu}

\author{
By Tim Fulmer, Senior Writer
}

Researchers at the Mount Sinai School of Medicine have used microRNA to generate influenza strains that are attenuated in humans but not in chicken eggs. ${ }^{1}$ The strategy could potentially lead to safe and effective live attenuated flu vaccines with high manufacturing yields.

Although live attenuated vaccines have multiple efficacy advantages over inactivated vaccines, the technology for attenuation hasn't changed in the past 40 years and relies on spontaneous mutagenesis under suboptimal conditions to create a suitable strain. The Mount Sinai researchers were looking to rationalize and speed up the process, and now they have shown that miRNA can be used to generate viruses with species-specific attenuation. ${ }^{1}$

Lack of attenuation in chicken eggs, in which flu vaccines are currently produced, is important for high production yields, whereas some level of attenuation in humans is essential for safety.

miRNAs are 23-nucleotide single-stranded RNAs that control gene expression by binding to sequences in a target mRNA and repressing its translation. Engineering miRNA target sequences into a viral genome can thus alter or abolish expression of proteins, potentially reducing a virus' replication fitness and result-
"The fact that the genetic modification is in two defined positions on a single gene makes for a clean and simple quality assurance assay." -Alan Shaw, VaxInnate Corp. survived a lethal $\mathrm{H} 1 \mathrm{~N} 1$ or $\mathrm{H} 5 \mathrm{~N} 1$ challenge at 21 days postvaccination, whereas all mock-vaccinated controls showed rapid weight loss and died.

The findings were published in Nature Biotechnology.

"The paper's strategy of harnessing the host's cellular machinery to attenuate a virus stands in marked contrast to established methods that attenuate virus in some form of cell culture," said George Kemble, VP of R\&D and head of California operations at MedImmune Inc., a subsidiary of AstraZeneca plc that markets FluMist, the only approved live attenuated influenza vaccine.

The creation of the first FluMist strains used cold adaptation, an established method of viral attenuation. A live attenuated influenza strain was derived from a wild-type strain by serial passage in primary chick kidney cells at sequentially lower temperatures. During that process, the virus acquired mutations in various gene segments that produced a cold-adapted, temperature-sensitive and attenuated phenotype. $^{2}$

\section{Finding a silent balance}

The paper's findings also suggest that it might be easier to monitor unwanted genetic drift in an miRNA-attenuated strain than in a coldadapted strain, said Alan Shaw, president and CEO of vaccine company

VaxInnate Corp. "The fact that the genetic modification is in two defined positions on a single gene makes for a clean and simple quality assurance assay. That's in contrast to strains that are attenuated by selection for temperature sensitivity, where the attenuated phenotype is the result of multiple point mutations on different gene segments," he said.

Indeed, a master viral strain that serves as the backbone for seasonal FluMist can have as ing in an attenuated strain.

With this in mind, the Mount Sinai researchers looked for miRNAs that were ubiquitous in the lung tissue of humans and mice but absent in chickens. Computational analysis of miRNA profiles from the three species yielded miRNA-93 as a candidate.

The researchers then introduced two copies of the miRNA-93 target sequence into a highly conserved region within the gene encoding influenza viral nucleoprotein. They reasoned that a highly conserved region would be less likely to undergo spontaneous mutation and escape control by miRNA-93, which could cause an attenuated strain to revert to a pathogenic phenotype.

The group rationally modified strains of $\mathrm{H} 1 \mathrm{~N} 1$ and $\mathrm{H} 5 \mathrm{~N} 1$ with miRNA-93 and characterized levels of viral attenuation in eggs and mice.

In 10-day-old embryonated chicken eggs, the two modified strains were not attenuated and produced viral titers similar to those of their respective wild-type strains.

In contrast, the modified strains were attenuated in mice, and animals given the vaccination had negligible weight loss-a measure of pathogenicity - compared with uninfected mice. All vaccinated mice many as 10 different mutations in at least four different genes to generate an acceptable level of attenuation. ${ }^{2}$

However, Kemble cautioned that fewer mutations are not necessarily better when it comes to viral attenuation. "A larger number of mutations can provide a form of redundancy that ensures a strain's genetic stability and guards against reversion," he told SciBX. "Thus, for any live attenuation strategy, we're looking for a balance. The minimal number of mutations to properly attenuate must be balanced against a sufficient number of mutations to ensure the chances of reversion are astronomically small."

Benjamin tenOever, corresponding author on the Nature Biotechnology paper, told SciBX his lab is generating a panel of attenuated influenza virus strains to get a better idea of the number and placement of miRNA target sequences required for optimal vaccine safety and immunogenicity. tenOever is assistant professor of microbiology and a researcher in the Global Health and Emerging Pathogens Institute at Mount Sinai.

"Over the next couple of years we'll explore variations on the approach we describe in the paper. We'll look at using two or more different miRNA target sequences in a single segment of the viral genome 
or in multiple segments, and we'll also look at using multiple copies of the same miRNA target sequence in single or multiple segments. Thus, a variety of permutations can be tested and optimized," he said.

Adding other miRNAs in addition to the species-specific one described in the paper could serve to attenuate the virus in a tissuespecific manner, said Kalle Saksela, professor and chairman of the Department of Virology at the University of Helsinki. If the virus were specifically attenuated in host tissues that show the most pathology, like the respiratory tract in the case of influenza, a strong immune response would nevertheless occur while reducing the likelihood that virus surviving in other tissues could revert to a more pathogenic strain, he said.

In 2008, Saksela and collaborators at the University of California, San Francisco, reported using an miRNA-based approach to generate a tissue-specific live attenuated poliovirus strain that replicated in non-neuronal tissues but not in the CNS. That strain elicited high levels of neutralizing antibodies without causing disease and protected mice against a lethal challenge of wild-type poliovirus. ${ }^{3}$

Saksela did not disclose his plans for the vaccine, though he and colleagues will continue to use the miRNA-based approach to create other viral strains that are attenuated in a tissue-specific manner.

Regardless of which miRNAs are used, Kemble recommended characterizing the attenuated strains in additional preclinical models. "The strategy clearly attenuates virus in mice. A logical next step is looking at disease outcomes of the attenuated strains in animals that more closely mimic human flu infection, including ferrets and perhaps even nonhuman primates," he said.

"We'll begin in mice, where we can initially assess toxicity and immunogenicity," said tenOever. "Strains that elicit high levels of neutralizing antibodies and are also properly attenuated-as judged by minimal weight loss and fever following infection-will then be further tested in ferrets."

\section{Enhancing yield}

On the manufacturing front, tenOever's group hopes to switch from egg-based production of their attenuated vaccines to a cell-culture system. The goal is to reduce the 3-6 months required to produce substantial quantities of a new vaccine strain in eggs.

In addition, said tenOever, "a cell-culture system would make vac- cine manufacture less dependent on the supply of chicken eggs, which can become vulnerable during avian flu outbreaks, and also yield a vaccine safe for people with egg allergies."

Even in eggs, VaxInnate's Shaw thinks the miRNA-attenuated strains could produce higher viral yields compared with other attenuation methods. The propagation of an miRNA-based attenuated strain in the standard egg-based system might be enhanced by growing the modified strains at $37^{\circ} \mathrm{C}$ rather than at the lower temperatures required for cold-adapted attenuated flu strains like FluMist, he said.

Indeed, in a small pilot experiment, tenOever and colleagues found that an miRNA-attenuated strain grown at $37^{\circ} \mathrm{C}$ produced $9-12 \mathrm{~mL}$ of allantoic fluid per egg. By contrast, a FluMist strain produced an average of $7-10 \mathrm{~mL}$ at $33^{\circ} \mathrm{C}$, the temperature at which that strain was no longer attenuated and thus able to replicate. Allantoic fluid is the portion of the egg that is harvested for viral particles.

Given that allantoic fluid volume is proportional to the number of infective virus particles, "that would suggest our species-specific attenuation allows for as much as $70 \%$ more vaccine per egg," tenOever said.

A patent application has been filed covering the incorporation of species-specific miRNA target sequences into the coding regions of viral genomes to control viral tropism and viral replication. The findings are available for licensing from the Mount Sinai School of Medicine's Office of Technology and Business Development.

Fulmer, T. SciBX 2(24); doi:10.1038/scibx.2009.963

Published online June 18, 2009

\section{REFERENCES}

1. Perez, J. et al. Nat. Biotechnol.; published online May 31, 2009; doi:10.1038/nbt.1542

Contact: Benjamin tenOever, Mount Sinai School of Medicine, New York, N.Y.

e-mail: benjamin.tenoever@mssm.edu

2. Ambrose, C. et al. Influenza Other Respi. Viruses 2, 193-202 (2008)

3. Barnes, W. et al. Cell Host Microbe 4, 239-248 (2008)

COMPANIES AND INSTITUTIONS MENTIONED

AstraZeneca plc (LSE:AZN; NYSE:AZN), London, U.K.

MedImmune Inc., Gaithersburg, Md.

Mount Sinai School of Medicine, New York, N.Y.

University of California, San Francisco, Calif.

University of Helsinki, Helsinki, Finland

VaxInnate Corp., Cranbury, N.J. 